\title{
Article
}

\section{"To hit, or not to hit?" Examining the similarity between practice and real swings in golf}

Carson, H.J., Collins, D., and Richards, J.

Available at http://clok.uclan.ac.uk/12091/

Carson, H.J. ORCID: 0000-0002-3785-606X, Collins, D., ORCID: 0000-00027601-0454 and Richards, J. ORCID: 0000-0002-4004-3115 (2014) "To hit, or not to hit?" Examining the similarity between practice and real swings in golf. International Journal of Golf Science, 3 (2). pp. 103-118. ISSN 2168-7595

It is advisable to refer to the publisher's version if you intend to cite from the work. http://dx.doi.org/10.1123/ijgs.2014-0003

For more information about UCLan's research in this area go to http://www.uclan.ac.uk/researchgroups/ and search for <name of research Group>.

For information about Research generally at UCLan please go to http://www.uclan.ac.uk/research/

All outputs in CLoK are protected by Intellectual Property Rights law, including Copyright law. Copyright, IPR and Moral Rights for the works on this site are retained by the individual authors and/or other copyright owners. Terms and conditions for use of this material are defined in the policies page.

\section{CLoK}

Central Lancashire online Knowledge www.clok.uclan.ac.uk

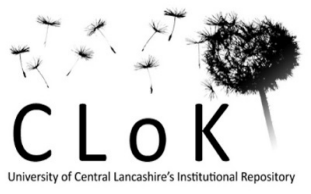


This is a pre-proof corrected manuscript as accepted for publication, of an article published in International Journal of Golf Science, CHuman Kinetics, in December 2014, available online: http://journals.humankinetics.com/ijgs-back-issues/ijgs-volume-3-issue-2december/to-hit-or-not-to-hit-examining-the-similarity-between-practice-and-real-swings-ingolf

7

“To Hit, or Not to Hit?" Examining the Similarity between Practice and Real Swings in

*Correspondence concerning this paper should be addressed to Howie J. Carson,

Institute of Coaching and Performance, University of Central Lancashire, Preston, United

27 Kingdom, PR1 2HE.

E-mail: hcarson1@uclan.ac.uk 
Abstract

30

31

32

33

34

35

36

37

Practice swings are commonly employed among golfers, presumably based on the tacit assumption that they share common psychomotor processes with real swings; however, this has not been verified by empirical research. Therefore, this study aimed to examine whether practice swings shared equivalent levels of control to real golf swings, when attempting the same target behavior. Three PGA Professional golf coaches and six amateurs (mean handicap $=2.7, \mathrm{SD}=2.2$ ) each executed 20 swings under two quasirandom conditions; 10 real swings when striking a ball and 10 practice swings without. Underpinned by the theoretical suggestions of the UnControlled Manifold (UCM) approach (Scholz \& Schöner, 1999), motor control was assessed using intraindividual movement variability. Results showed the level of equivalence to be inconsistent on both an inter and intraindividual basis. Coaches should, therefore, recognize that practice swings do not share the same effect for every golfer. Optimal coaching needs to consider individual responses before committing to specific training designs if counterproductive training is to be avoided.

Keywords: Coaching practice, movement variability, focus of attention, motor control, individual differences, imagery. 
“To Hit, or Not to Hit?" Examining the Similarity between Practice and Real Swings in Golf For coaches seeking to optimize the practice design of their pupils, many different factors need to be addressed. From a psychomotor perspective, previous research has shown how differences in the sequencing (blocked vs. random) and temporal distribution (massed vs. distributed) of practice (Goode \& Magill, 1986; Lee \& Genovese, 1988), feedback provision (Lee \& Carnahan, 1990), and model characteristics conveyed within a demonstration (Ste-Marie et al., 2012), can be controlled by coaches to enhance performance and the acquisition of motor skills over either short- or long-term timescales (Schmidt \& Bjork, 1992). Underpinning these coaching practices, or "tools," is the influence on performers' attentional control, which serves a critical role in the organization and efficiency of technique execution (Eysenck, Derakshan, Santos, \& Calvo, 2007). With this knowledge, coaches should be able to apply these tools when implementing, for instance, systems of skill acquisition (Fitts \& Posner, 1967) or refinement (Carson \& Collins, 2011); which are theoretically underpinned by the dynamic nature of attention. Crucially, therefore, not only must coaches be able to select appropriate tools at certain times within these systems, they must also be able to evaluate the resultant effect on performers' levels of attention and motor control. Unfortunately, this process is rarely "black and white" when designing individuallytailored practices; due to even subtle individual differences, one size does not always fit all (cf. MacPherson, Collins, Graham-Smith, \& Turner, 2013; Newell, Liu, \& Mayer-Kress, 2005). If, however, the effects of coaching tools were evaluated on an individual-basis and data provided pertained to psychomotor processes, coaches would be better able to plan, implement, reinforce, and/or modify their interventions for optimal effectiveness.

Despite this recognized requirement for individualized practice design, there is one tool which appears as almost ubiquitously employed by coaches and their golfers; namely, the practice swing, an overt physical simulation of an often impending movement: a full 
power swing without a ball. Within the literature, practice swings have been reported amongst golfers when performing a warm up (Fradkin, Finch, \& Sherman, 2001), attempting to make technical refinements (Carson, 2014), and as part of a preperformance routine intended to optimize skill execution (Cotterill, Sanders, \& Collins, 2010). Within professional coach education, practice swings have explicitly been promoted; as demonstrated by the following extract from a coaching manual of The Professional Golfers' Association of Great Britain and Ireland:

When you consider that a golf swing lasts less than 2 seconds, hitting 100 balls with this method [blocked practice] constitutes less than 3mins 40's worth of actual practice. With this in mind it is a good idea to carry out blocked practice without a ball as well as with a ball. Carrying out practice swings is as effective as hitting golf balls when using blocked practice. (PGA, 2008, p. 61)

In consideration of this common behavior, there is a tacit assumption that it shares common psychomotor processes as a real swing, otherwise why do it? Unfortunately, however, there is no scientific literature, to the best of our knowledge, which specifically addresses the similarity between these two versions of execution. Accordingly, the implementation of practice swings must be confirmed as equivalent by empirical investigation if consistency of a particular technique is the task goal (i.e., practice intended for a positive perturbation). Assessing the equivalence between practice and real golf swings can be undertaken on a number of different levels of system (i.e., performer) organization (cf. Newell, Liu, \& Mayer-Kress, 2001). Since this paper concerns the level of motor control, measures should relate to the processes through which execution depends; what Newell et al. term "microphenomena" (p. 63). Reflecting recent advances from dynamical systems theory, intertrial movement covariability has been shown to reveal the functional role of mechanical degrees of freedom (DoFs) which contribute to the control of technique. Scholz and Schöner 
(1999) proposed the UCM concept, that an abundance of DoFs within the motor system results in some being controlled (stable) and others uncontrolled (flexible; cf. Bernstein, 1967). Stable DoFs are identified by low levels of intertrial variability, whereas flexible DoFs demonstrate much high levels; crucially, however, the low level variability variables seem to be those most crucial to effective executions of the target task. By employing this method, Scholz and Schöner demonstrated that the center of mass position in the sagittal plane was more stable when compared to either hand or head position when executing a sitto-stand task. Consequently, this approach is able to attribute a level of importance given to individual technical components by the central nervous system towards achieving a desired task goal. The combination of stable and flexible components ensures reproduction of movement form (characterized by stable DoFs) while accommodating for unplanned perturbations imposed during execution (involving the flexible DoFs). Accordingly, once a skill is learned, intertrial movement variability should "settle down" to a consistent and functional, although individually-specific, level across the different DoFs and be maintained from session-to-session, with more "important" factors displaying lower levels of variability. As a novel extension of the UCM approach, Carson, Collins, and Richards (2014) suggested that the same nonlinear pattern of movement covariability could result from the manipulation of performers' attentional focus. This would, therefore, provide an informative measure of dynamic attentional control during the process of skill refinement (cf. Carson \& Collins, 2011). Specifically, it was hypothesized that when a “. . .performer decides to work on a particular aspect of that movement by exerting increased conscious control, that particular part becomes more consistent (with even lower variability) whilst the variability of other nonassociated parts increases" (p. 330). Supporting this hypothesis, data were presented to demonstrate this effect when PGA Professional Golf Coaches made conscious short-term refinements to their techniques within a single session. Interpreting these findings 
against the theoretical percepts of the UCM approach, increased conscious control over a single DoF serves to increase its relative importance and lessen that of other technical components. In summary, the studies by Scholz and Schöner (1999) and Carson et al. (2014) reveal intertrial movement variability to reflect the functional organization of motor control, but which is also related to both conscious and subconscious cognitive processes. Crucially, application of this concept under differing task constraints (Newell, 1986) could be employed as a coaching tool to augment a coach's understanding of, and ability to evaluate, different methods of practice. Accordingly, practices designed to elicit equivalent psychomotor processes should reveal the same measure of intertrial movement variability across mechanical DoFs; for instance, when performing practice and real golf swings.

Indeed, such checks would seem essential if counterproductive (dysfunctional perturbation) training methods are to be avoided. Therefore, the aim of this study was to examine whether practice swings shared equivalent levels of control to real golf swings, when attempting the same target behavior. To reduce the chance of between condition differences, executions were performed by skilled golfers with already well-established techniques. We should stress that some level of variability within each condition is acceptable, perhaps even functional, but that excessive variability is clearly dysfunctional (cf. Gentile, 1972).

\section{Method}

\section{Participants}

Reflecting the need for advanced skill status, participant eligibility required no current injury (assessed through self-report) and a handicap of less than five. Accordingly, nine right-handed male golfers (A-I) between the ages of 17 and 44 years $(M=26.1, S D=8)$ were recruited for this study. Playing ability included PGA Professional Golf Coaches $(n=3$; no handicap however all held a maximum handicap of 4 upon turning professional) and amateur golfers $(n=6$; mean average handicap $=2.7, S D=2.2$ ). 


\section{Procedure}

Preceding data collection, participants were required to read an information sheet and provide signed informed consent. Ethical approval was granted from the University's Ethics Committee prior to data collection. Participants were randomly assigned the order of conditions; execution by striking a ball, the "ball condition", followed by practice swings, the "practice swing condition", or vice versa.

To minimise the potential for any warm up effect, participants were allocated as much time as required to warm up. Accordingly, the warm up period ceased when each participant conveyed verbally that they were ready to commence with the testing. Warm ups were typified by the use of self-conducted stretching exercises, practice swings, and shots using participants' own 7-iron and legally conforming golf balls. A 7-iron was selected for use during this study because it is a commonly used club during play and practice conditions; consequently, it would likely represent a skill that was well-established.

Participants' body dimensions were measured, including; body height, arm span (distal end of the right hand's middle finger to the distal end of the left hand's middle finger when adopting a " $\mathrm{T}$ " pose), hip height (ground to the most lateral bony prominence of the greater trochanter) and width (right to left anterior superior iliac spine), and shoulder width (right to left distal tip of acromion). Following, participants were fitted with an inertialsensor motion capture suit (MVN Biomech Suit, Xsens ${ }^{\circledR}$ Technologies B.V., The Netherlands). Sensors were affixed to segment landmarks on the pelvis (flat on the sacrum), shoulders (scapulae), and sternum (proximal end) using Velcro strapping, the hands using fitted gloves (above the metacarpals), and the head using a head band (superior and posterior to the right ear) in accordance with the manufacturer's guidelines. Following, a second warm up phase provided familiarity and comfort in wearing the suit, and allow any necessary adjustments to the strapping to be made. The motion capture suit was then calibrated to 
determine joint centers of each participant, incorporating the earlier measured body dimensions. This was performed by employing a "neutral" static, followed by dynamic handtouch calibration process whereby, the sensor to segment alignment and segment lengths are estimated by solving the closed kinematic chain for each pose. In addition, a single trial was captured when adopting the anatomical position to allow an anatomical model to be created. Depending on the randomly assigned test condition order, participants executed 10 full swings using their own 7-iron in either the ball or practice swing condition; followed by another 10 swings to satisfy the alternative condition. To increase levels of adherence towards executing the same target behavior, participants were reminded following Trials 3,6 , and 9 of each condition to try and achieve a typical technique and distance that they would normally perform during play. Given the likelihood of each participant's target behavior being idiosyncratic; it was inappropriate to provide a specific technical or mental instruction which would, of course, have a differential level of impact. As such, in order to assess any mentally induced differences in movement variability for the same target behavior, it was important to allow a natural and individually preferred response to the task. Executions were conducted from an artificial golf mat into an indoor net $15 \mathrm{~m}$ away, aiming for the same target each time - a vertical line running the entire height of the net. Maintaining a consistent hitting surface provided an enhanced level of experimental control. All kinematic data were collected using a sampling rate of $120 \mathrm{~Hz}$.

\section{Data Processing and Analysis}

Raw data from the MVN Studio software (Xsens ${ }^{\circledR}$ Technologies B.V., The Netherlands) were exported into c3d file format and analyzed with Visual3D ${ }^{\mathrm{TM}} \mathrm{v} 4.89 .0$ software (C-Motion ${ }^{\circledR}$ Inc., Germantown, MD, USA) using six DoFs modeling. Three events were automatically identified and used to divide the swing into two phases, the backswing and downswing. The first event, "swing onset," was defined as the frame when the left 
hand's center of gravity linear velocity crossed a threshold value of $0.2 \mathrm{~m} / \mathrm{s}$ in the local medial-lateral axis relative to the pelvis. The second event, "top of swing," was defined as the frame when the right hand distal end position reached its maximum value in the global vertical axis prior to the third event occurring. The third event, "bottom of swing," was defined as the frame when the distal end position of the right hand reached its minimum position in the global vertical axis. Accordingly, bottom of swing represented the "end event"; no data were included for the remainder of the swing. Following, the time between each event was normalized to 101 points.

In consideration of the study's aim, an analysis of every kinematic variable was not possible. As such, the left hand position was referenced to the local co-ordinate system of the sternum in three-dimensions (3D) as a representative variable. This variable was selected because it was believed to provide a good reflection of the swing principle width of arc, which is defined by professional golf coaching texts in terms of the relationship between the lead (left in right handed golfers) hand and center of golf swing rotation. According to Nesbit and McGinnis (2009), the radius path of the hand during the swing influences the kinetic loading on the golfer and therefore the transfer of kinetic energy to the club. Optimizing the hand path was thus shown to demonstrate increases in club head velocity; a factor which is primarily associated with increased shot distance (Sweeney, Mills, Alderson, \& Elliott, 2013). Figure 1 represents the width of arc using a two-dimensional (2D) image in the global coronal plane - the referenced standard for golf coaching practice. Accordingly, in this study, the medial-lateral, anterior-posterior, and superior-inferior hand position relative to the sternum were exported to Microsoft Excel $^{\circledR} 2010$ and standard deviations for all 101 points between each event during the two conditions were plotted for each participant (cf. Carson et al., 2014).

\section{Results}


Data are shown in Figures 2, 3, and 4 which represent the intertrial movement variability of the medial-lateral, anterior-posterior, and superior-inferior position of the left hand to sternum position for participants across the two different conditions. Visual inspection of these graphs reveal the highly individual-nature of effect between the ball and practice swing conditions; executing practice swings did not have the same influence on all participants. Therefore, findings pertaining to the level of equivalence between conditions on an intraindividual basis are reported below. Note however, that the themes within the findings are also applicable as interindividual comparisons. For clarity and to highlight specific aspects of the analyses, exemplar participant graphs are referred to throughout, with individual qualitative summaries provided in Table 1.

Results reveal a number of findings with regards to the equivalence between conditions; clearly data are highly complex. Firstly, data show temporal inconsistencies within the swing for many participants. For instance, Participant F (Figure 2) demonstrates three moments during the swing where variability levels are noticeably separated: at the swing onset, $50 \%$ during the backswing, and $90 \%$ during the downswing. Participant $\mathrm{E}$ (Figure 3) shows a consistent discrepancy for most of the swing up until $70 \%$ during the downswing. Whereas, Participant D (Figure 4) shows greater equivalence for the downswing compared to the backswing.

In addition, individuals showed differences in the level of equivalence between the planes of motion. As exemplified by Participant G, showing what we would consider to be a consistent and reasonably good level of equivalence for the majority of the swing in the medial-lateral (Figure 2) and anterior-posterior (Figure 3) planes of motion; however, this is less well-reflected in the superior-inferior (Figure 4) plane. Likewise, Participant A shows a similar effect across the same planes of motion. For Participant E, data in the medial-lateral 
254

255

256

257

258

259

260

261

262

263

264

265

266

267

268

269

270

271

272

273

274

275

276

277

278

(Figure 2) and superior-inferior (Figure 4) planes of motion show a largely equivalent amount of variability, which is not shared by the anterior-posterior (Figure 3) plane data.

Finally, the disparity of variability between the two conditions was not always consistent in its "direction" across the planes of motion. That is, sometimes the practice swing condition demonstrated a higher level of variability compared to the ball condition. For example, Participant I shows a consistently increased level of variability for the ball condition in the medial-lateral (Figure 2) and superior-inferior (Figure 4) planes of motion, but the opposite effect in the anterior-posterior (Figure 3) plane. Whereas, Participants D and $\mathrm{H}$ showed a predominantly increased amount of variability in the ball condition compared to the practice swing condition in all three planes of motion.

\section{Discussion}

The aim of this study was to examine whether practice swings shared equivalent levels of control to real golf swings, when attempting the same target behavior. The overall result showing differences in effect between participants is perhaps unsurprising, since interventions are dependent on each performer's “dynamic state” (Newell et al., 2005, p. 46): a reference to the developed control processes underpinning the skill of each performer. From an applied perspective, the important implication for coaches, at least when working with low handicap golfers, is that employing practice swings will not impact on every golfer in the same way. Data from this study reveal the subtle interparticipant differences that exist between the two conditions; the answer to knowing whether or not to employ practice swings is certainly not black or white. Indeed, this finding supports several other intraindividual analyses in sport which have questioned the veracity of "received wisdom" when coaching high-level athletes. For example, MacPherson et al. (2013) demonstrated four out of six elite-level horizontal jumpers to perform their upper quartile performances when the pattern of footfall variation was consistently lower for 15 strides prior to contact with the takeoff 
board, when compared to the lower quartile performance which were much higher in variability across the 15 strides. This is in contrast to received wisdom suggesting that variability should reduce from only five strides prior to takeoff (cf. Lee, Lishman, \& Thomson, 1982). Likewise, in archery and many target-oriented sports, it is commonly assumed that heart rate deceleration immediately prior to arrow release is predictive of optimal performance (Tremayne \& Barry, 2001). However, on inspection of individual data from elite-level archers, this pattern was not apparent across all (Collins, 2002).

Consequently, at present, we suggest caution towards the ubiquitous employment of practice swings if the aim is to enhance subsequent execution and avoid a negative transfer effect where, for some golfers, the latter would seem to be a genuine possibility. Therefore, optimal coaching practice should be viewed as that which attends to the response of each performer on an individual-basis, prior to committing to specific training designs.

From the perspective of the UCM approach (cf. Scholz and Schöner, 1999), the variability graphs (Figures 2-4) imply that movement was differentially organized both between and within the two conditions; the central nervous system dynamically altered the amount of importance allocated to each of the DoFs. Furthermore, reflecting the findings of Carson et al. (2014), differences between conditions could have resulted from inconsistent patterns of cognition. We present no data in this paper to demonstrate such a cause; however, our initial speculation is that this might have been an underlying and influential factor to explaining the results. On the basis that Carson et al. showed a consistent change in the amount of movement variability under contrasting conditions of attentional focus, such speculation should be considered as supported by reasoned evidence. If practice swing effectiveness as a preperformance prime is dependent on a performer's cognitions, it is worth addressing possible tools that might help "equip" performers to optimize their practice design. Previous applied and theoretical research has 
strongly supported the beneficial employment of multimodal imagery as a tool for accurately activating neural networks involved in movement execution (e.g., Collins, Morriss, \& Trower, 1999; Holmes \& Collins, 2001; MacPherson, Collins, \& Obhi, 2009); what cognitive psychologists would refer to as memory retrieval. As such, those participants who were better able to execute under both conditions by attending to the same sensory stimuli, would be more likely to demonstrate equivalent levels of control. Adopting a similar attentional strategy could also be interpreted as a reflection on participants' levels of intent during movement organization and execution; therefore suggesting the requirement for a sufficient level of psychological skill in order to benefit from employing practice swings. If this were to be the case, the mixed results in this study would be supported by the inconsistent use of psychological skills previously reported by golfers (Carson, Collins, \& MacNamara, 2013). Clearly future work is required to verify this possible link. Were this research to find strong causality however, it would present a robust case for the implementation of psychological skills training in parallel with executing practice swings, for those performers showing low levels of equivalence between the two conditions.

Notwithstanding the advances that have been made to understanding the optimization of practice, this study was not without limitation. For instance, psychometric data pertaining to imagery ability were not collected. Completion of the Vividness of Movement Imagery Questionnaire-2 (VMIQ-2; Roberts, Callow, Hardy, Markland, \& Bringer, 2008) or Sport Imagery Ability Questionnaire (SIAQ; Williams \& Cummings, 2011) could have validated our speculation that imagery ability is a causative factor of equivalence between practice and real swings. Another limitation of this study relates to the ecological validity of test conditions, although it should be recognised that it is not uncommon for golfers to practice in front of a net. It is also not known whether our findings are valid across different skill levels 
of golfer, or indeed other swing variables that are unrelated to the left hand relative to the sternum position.

To overcome limitations within this study, we propose several directions for future research. Firstly, the suggestion that imagery might moderate the similarity between practice and real swings could be explored using supplementary psychometric assessment. Secondly, the differences between blocked and interleaved trials of real and practice swings should be explored, as it is not known whether our blocked approach could have influenced the findings. Thirdly, collecting data in more ecologically valid environments could offer further insight. Indeed, such inclusion would be supported by theory (Lang, 1979), since greater congruence between stimulus propositions would be apparent, and recommended guidelines for practicing mental imagery (Holmes \& Collins, 2001). For research purposes, this might consist of hitting shots and performing practice swings in front of a golf simulator. Finally, research should seek to explore whether genuine improvements in imagery ability, following theoretically grounded intervention, are better able to reduce the discrepancy between practice swings and real swings and assess the impact on subsequent performance (both outcome and consistency), that is, skill level.

In conclusion, by employing intraindividual movement variability as a tool for assessing motor control, this study showed practice swings to share different amounts of equivalence with real swings, despite similarity of skill status between golfers. As such, we hope to have raised awareness amongst golf coaches against the implementation of a "one size fits all" approach when designing optimal training tasks. While much research is required to develop a more complete understanding of how best to employ practice swings, this study represents an initial step to being able to ask fundamental questions about their use. 


\section{References}

354

355

356

Bernstein, N.A. (1967). The coordination and regulation of movements. Oxford: Pergamon Press.

Carson, H.J. (2014). Working inside the black box: Refinement of pre-existing skills (Unpublished doctoral Dissertation). University of Central Lancashire, Preston, England. Retrieved from http://clok.uclan.ac.uk/9602/

Carson, H.J., \& Collins, D. (2011). Refining and regaining skills in fixation/diversification stage performers: The Five-A Model. International Review of Sport and Exercise Psychology, 4, 146-167. doi:10.1080/1750984X.2011.613682

Carson, H.J., Collins, D., \& MacNamara, Á. (2013). Systems for technical refinement in experienced performers: The case from expert-level golf. International Journal of Golf Science, 2, 65-85.

Carson, H.J., Collins, D., \& Richards, J. (2014). Intra-individual movement variability during skill transitions: A useful marker? European Journal of Sport Science, 14, 327-336. doi:10.1080/17461391.2013.814714

Collins, D. (2002). Psychophysiology and sports performance. In B. Blumenstein, M. BarEli, \& G. Tenenbaum (Eds.), Brain and body in sport and exercise (pp. 15-36). Chichester: Wiley.

Collins, D., Morriss, C., \& Trower, J. (1999). Getting it back: A case study of skill recovery in an elite athlete. The Sport Psychologist, 13, 288-298.

Cotterill, S.T., Sanders, R., \& Collins, D. (2010). Developing effective pre-performance routines in golf: Why don't we ask the golfer? Journal of Applied Sport Psychology, 22, 51-64. doi:10.1080/10413200903403216 
Eysenck, M.W., Derakshan, N., Santos, R., \& Calvo, M.G. (2007). Anxiety and cognitive performance: Attentional control theory. Emotion 7, 336-353. doi: $10.1037 / 15283542.7 .2 .336$

Fitts, P.M., \& Posner, M.I. (1967). Human performance. California: Brooks/Cole Publishing Company.

Fradkin, A., Finch, C., \& Sherman, C. (2001). Warm up practices of golfers: Are they adequate? British Journal of Sports Medicine, 35, 125-127. doi:10.1136/bjsm.35.2.125

Gentile, A.M. (1972). A working model of skill acquisition with application to teaching. Quest, 17, 3-23. doi:10.1080/00336297.1972.10519717

Goode, S., \& Magill, R.A. (1986). Contextual interference effects in learning three badminton serves. Research Quarterly for Exercise and Sport, 57, 308-314. doi:10.1080/02701367.1986.10608091

Holmes, P.S., \& Collins, D.J. (2001). The PETTLEP approach to motor imagery: A functional equivalence model for sport psychologists. Journal of Applied Sport Psychology, 13, 60-83. doi:10.1080/10413200109339004

Lang, P.J. (1979). A bio-informational theory of emotional imagery. Psychophysiology, 16, 495-512. doi:10.1111/j.1469-8986.1979.tb01511.x

Lee, D.N., Lishman, J.R., \& Thomson, J.A. (1982). Regulation of gait in long jumping. Journal of Experimental Psychology. Human Perception and Performance, 8, 448459. doi:10.1037/0096-1523.8.3.448

Lee, T.D., \& Carnahan, H. (1990). Bandwidth knowledge of results and motor learning: More than just a relative frequency effect. The Quarterly Journal of Experimental Psychology Section A, 42, 777-789. doi:10.1080/14640749008401249 
400

401

402

403

404

405

406

407

408

409

410

411

412

413

414

415

416

417

418

419

420

421

422

423

Lee, T.D., \& Genovese, E.D. (1988). Distribution of practice in motor skill acquisition: Learning and performance effects reconsidered. Research Quarterly for Exercise and Sport, 59, 277-287. doi:10.1080/02701367.1988.10609373

MacPherson, A.C., Collins, D., Graham-Smith, P., \& Turner, A.P. (2013). Using rhythmicity to promote performance in horizontal jumps: An exemplar of the need for intraindividual intervention. International Journal of Sport Psychology, 44, 93-110. doi:10.7352/IJSP.2013.44.093

MacPherson, A.C., Collins, D., \& Obhi, S.S. (2009). The importance of temporal structure and rhythm for the optimum performance of motor skills: A new focus for practitioners of sport psychology. Journal of Applied Sport Psychology, 21, 48-61. doi:10.1080/10413200802595930

Nesbit, S.M., \& McGinnis, R. (2009). Kinematic analyses of the golf swing hub path and its role in golfer/club kinetic transfers. Journal of Sports Science and Medicine, 8, 235246.

Newell, K.M. (1986). Constraints to the development of coordination. In M.G. Wade \& H.T.A. Whiting (Eds.), Motor development in children: Aspects of coordination and control (pp. 341-360). Dordrecht, The Netherlands: Martinus Nijhoff.

Newell, K.M., Liu, Y-T., \& Mayer-Kress, G. (2001). Time scales in motor learning and development. Psychological Review, 108, 57-82. doi:10.1037/0033-295X.108.1.57

Newell, K.M., Liu, Y-T., \& Mayer-Kress, G. (2005). Learning in the brain-computer interface: Insights about degrees of freedom and degeneracy from a landscape model of motor learning. Cognitive Processing, 6, 37-47. doi:10.1007/s10339-004-0047-6

PGA. (2008). Study guide: Golf coaching 2. United Kingdom: The Professional Golfers' Association. 
424

425

426

427

428

429

430

431

432

433

434

435

436

437

438

439

440

441

442

443

444

445

446

Roberts, R., Callow, N., Hardy, L., Markland, D., \& Bringer, J. (2008). Movement imagery ability: Development and assessment of a revised version of the Vividness of Movement Imagery Questionnaire. Journal of Sport and Exercise Psychology, 30, $200-221$.

Schmidt, R.A., \& Bjork, R.A. (1992). New conceptualizations of practice: Common principles in three paradigms suggest new concepts for training. Psychological Science, 3, 207-217. doi:10.1111/j.1467-9280.1992.tb00029.x

Scholz, J.P., \& Schöner, G. (1999). The uncontrolled manifold concept: Identifying control variables for a functional task. Experimental Brain Research, 126, 289-306. doi:10.1007/s002210050738

Ste-Marie, D.M., Law, B., Rymal, A.M., Jenny, O., Hall, C., \& McCullagh, P. (2012). Observation interventions for motor skill learning and performance: An applied model for the use of observation. International Review of Sport and Exercise Psychology, 5, 145-176. doi:10.1080/1750984X.2012.665076

Sweeney, M., Mills, P., Alderson, J., \& Elliott, B. (2013). The influence of club-head kinematics on early ball flight characteristics in the golf drive. Sports Biomechanics, 12, 247-258. PubMed doi:10.1080/14763141.2013.772225

Tremayne, P., \& Barry, R.J. (2001). Elite pistol shooters: Physiological patterning of best vs. worst shots. International Journal of Psychophysiology, 41, 19-29. doi:10.1016/S0167-8760(00)00175-6

Williams, S.E., \& Cummings, J. (2011). Measuring athlete imagery ability: The Sport Imagery Ability Questionnaire. Journal of Sport and Exercise Psychology, 33, 416440. 


\section{Qualitative Comparison}

\section{Participant}

Figure 2

Figure 3

Figure 4

A Consistently higher variability in the practice swing condition between $70 \%$ backswing and $80 \%$ downswing.

B Distinct fluctuation in variability at $40 \%$ backswing. Higher variability in the practice swing condition between $90 \%$ backswing and $45 \%$ downswing, then again from $90 \%$ downswing.

C Inconsistently higher variability in the ball condition until 30\% backswing. Inconsistently higher variability in the practice swing condition from $50 \%$ downswing.

D Inconsistently higher variability in the ball condition until $80 \%$ backswing.
Inconsistently higher variability in the practice swing condition from $70 \%$ downswing.

Generally consistent throughout, slightly lower variability in the practice swings condition until $50 \%$ backswing, slightly higher variability in the practice swing condition between $70 \%$ backswing and 55\% downswing.

Inconsistently higher variability in the practice swing condition from $25 \%$ backswing.

Inconsistently higher variability in the ball condition.
Inconsistently higher variability in the practice swing condition from $65 \%$ backswing.

Inconsistently higher variability in the practice swing condition between $75 \%$ backswing and $25 \%$ downswing, then again between $45-80 \%$ and from $90 \%$ downswing.

Consistently higher variability in ball condition from $50 \%$ backswing.

Inconsistently higher variability in the ball condition until $85 \%$ backswing and between 50-90\% downswing. 
E Consistently higher variability in the ball condition between $0-60 \%$ and $70-90 \%$ downswing.

Distinct fluctuation in variability at $60 \%$ backswing. Inconsistently higher variability in the ball condition from $80 \%$ downswing. Very similar amounts of variability between ball and practice swing conditions.
Inconsistently higher variability in the practice swing condition.

Inconsistently higher variability in the ball condition from $40 \%$ backswing.

Slight increase in variability in the practice swing condition between $55 \%-75 \%$ of backswing. Small and fluctuating changes in variability during the downswing.

Inconsistently higher variability in the ball condition until 55\% backswing, between $70 \%$ backswing and $70 \%$ downswing, the reverse occurred following $70 \%$ downswing.
Consistently higher variability in the ball condition between $85 \%$ backswing and $55 \%$ downswing. Inconsistently higher variability in the practice swing condition from $60 \%$ downswing.

Inconsistently higher variability in the practice swing condition between $40 \%$ backswing and $40 \%$ downswing.

Inconsistently higher variability in the ball condition between $60 \%$ backswing and $25 \%$ downswing. Inconsistently higher variability in the practice swing condition between 30-85\% downswing, the relationship reverses from $85 \%$ downswing.

Inconsistently higher variability in the ball condition $85 \%$ backswing. the practice swing condition until $65 \%$ backswing and $60-75 \%$ downswing. Inconsistently higher variability in the ball condition between $90 \%$ backswing and 55\% downswing and from $75 \%$ downswing. 
I Inconsistently higher variability in the ball condition between $35-80 \%$ backswing and $40-70 \%$ downswing.
Inconsistently higher variability in the ball condition until $70 \%$ backswing, inconsistently lower variability following $70 \%$ backswing.
Inconsistently higher variability in the ball condition between $20 \%$ backswing and $90 \%$ downswing. 


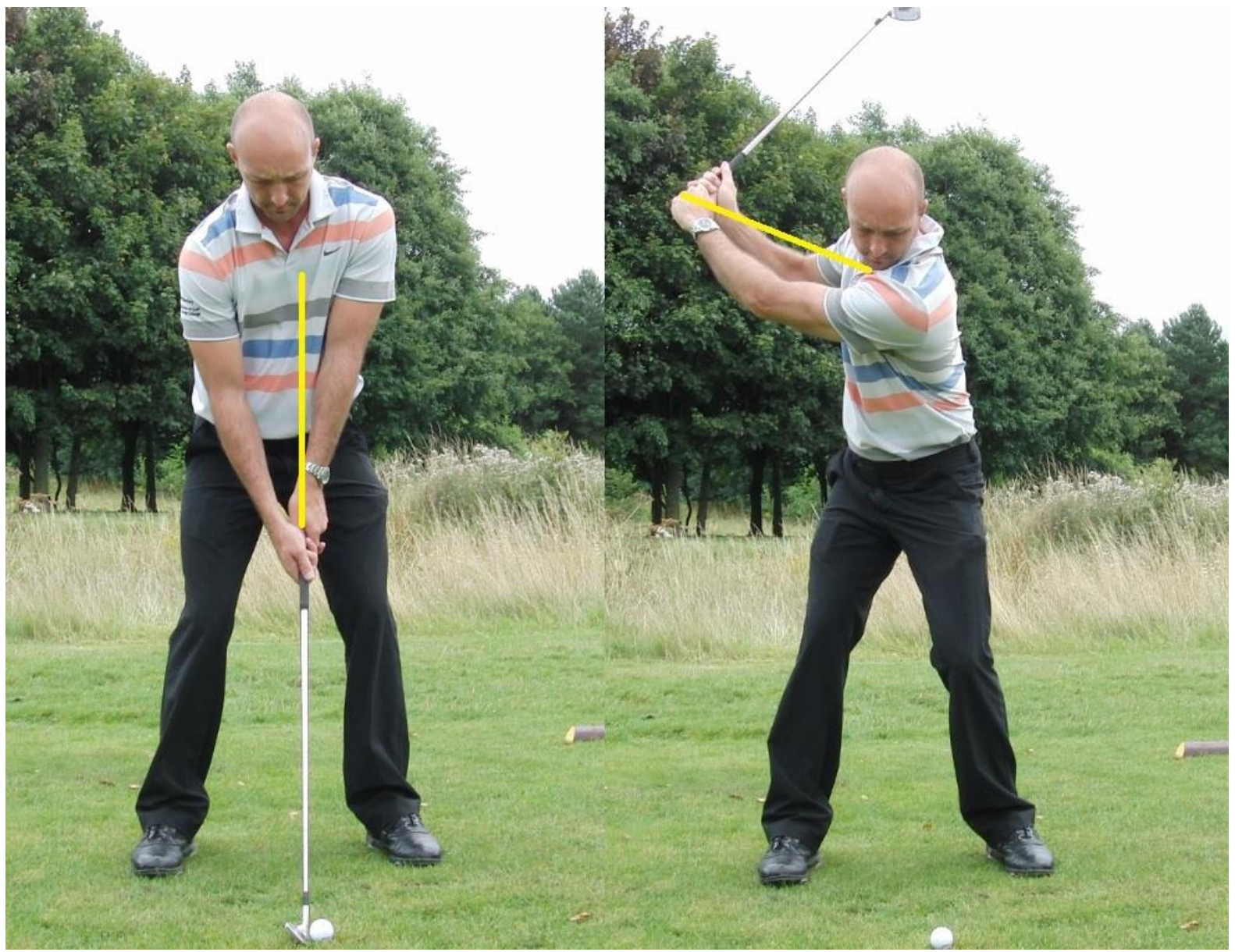

Figure 1. Width of arc defined by the distance between the hand and swing center, viewed at swing address (left) and at the top of swing (right).

452

453

454

455

456

457

458

459

460 
Running head: PRACTICE SWINGS IN GOLF

461

462

463

464

465

466

467

468

469

470

471

472

473

474

475

476

477

478

479

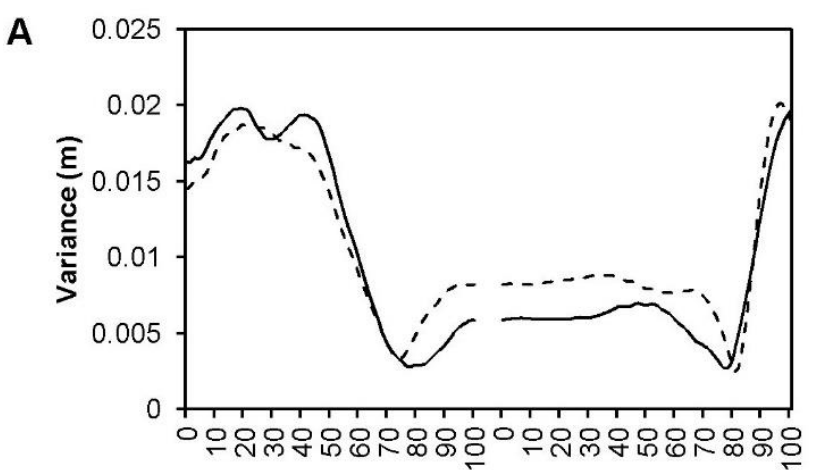

D

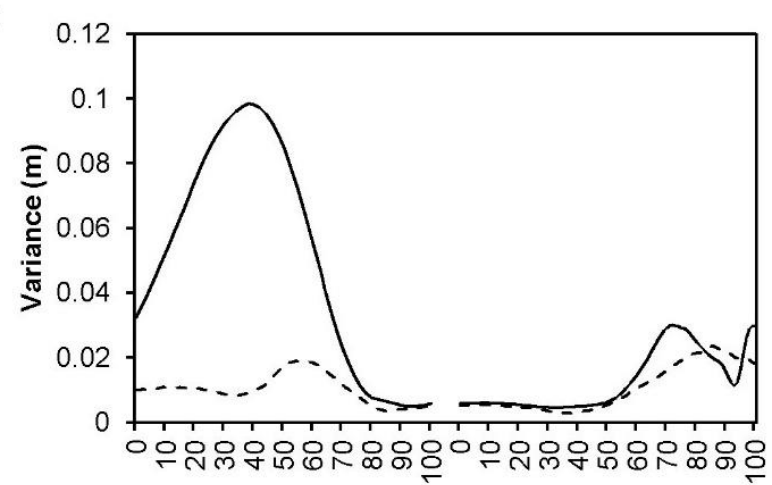

G

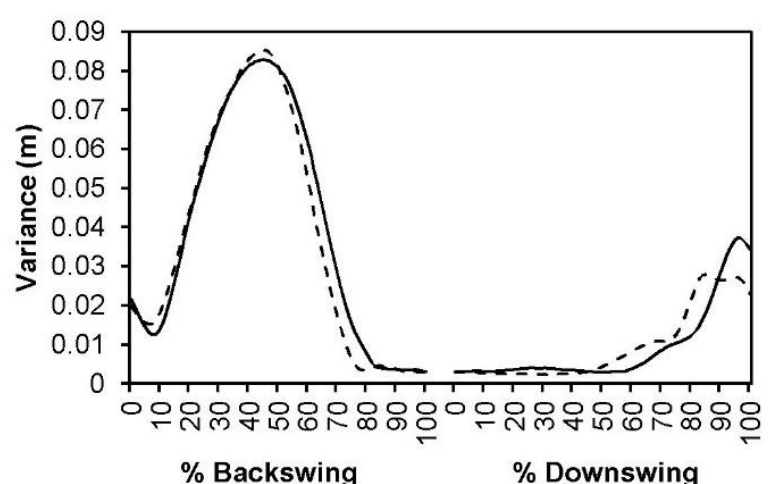
conditions.
B $\quad 0.02$

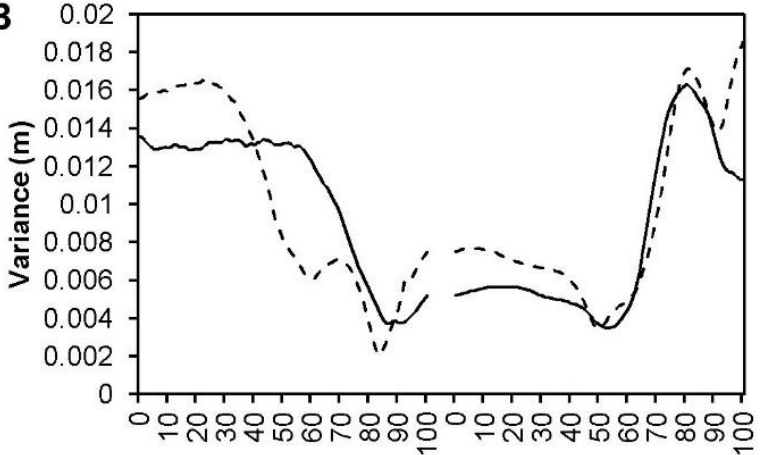

E $\quad 0.03$
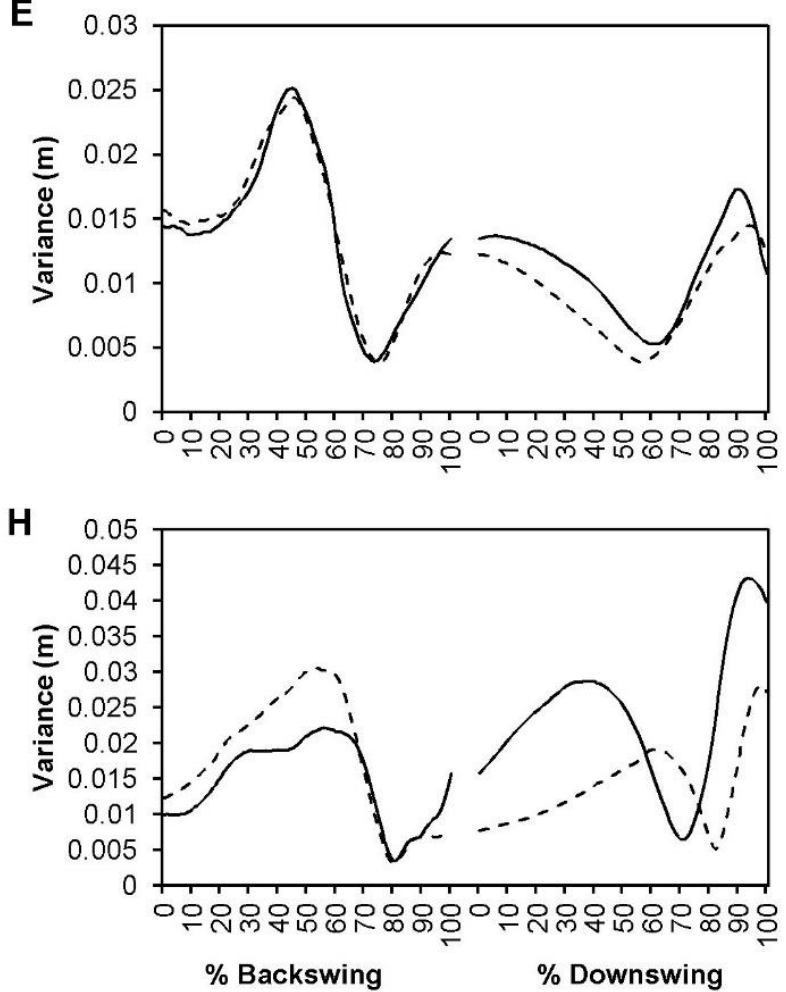
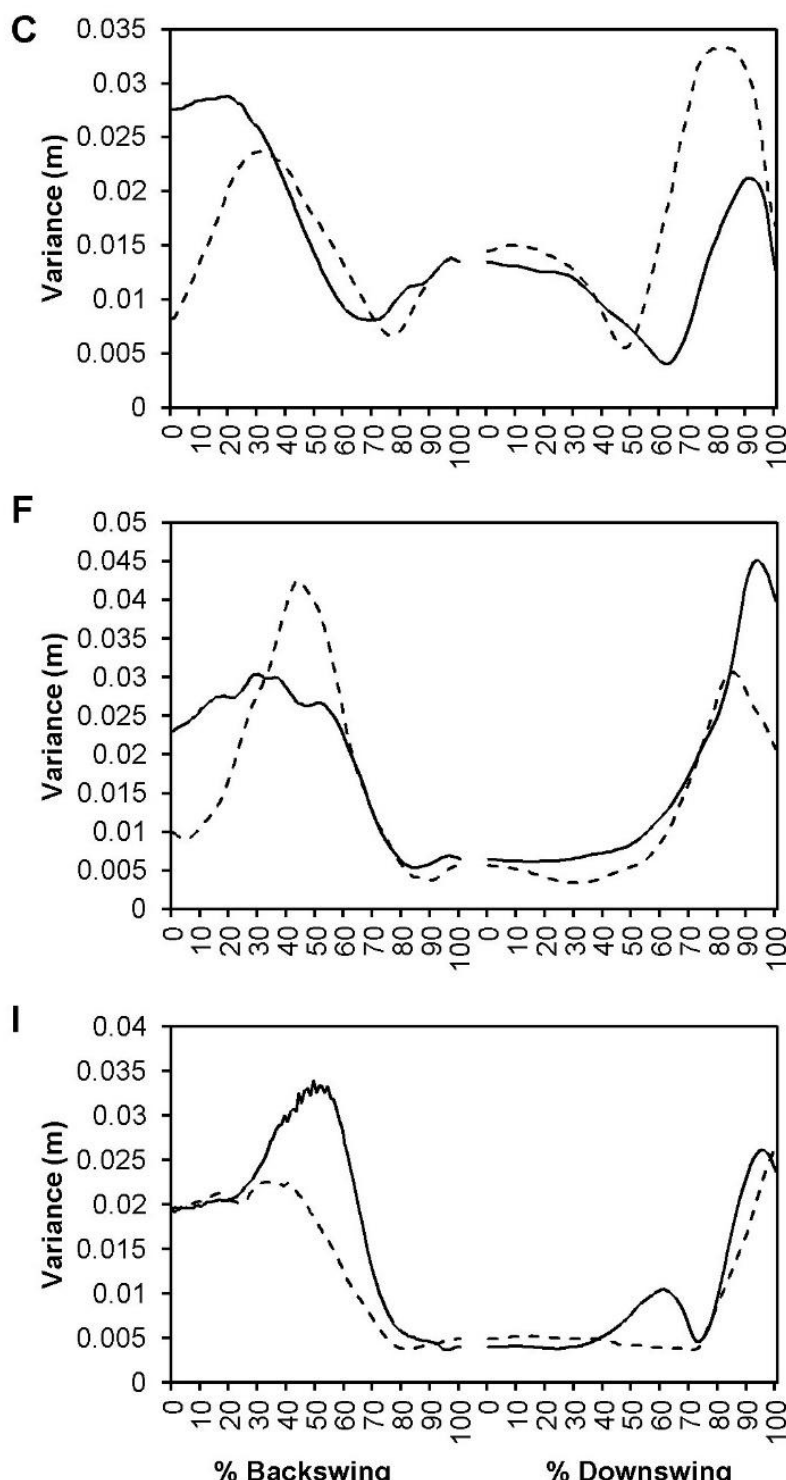

Figure 2. Intraindividual variability of left hand's medial-lateral position to the sternum for ball (solid line) and practice swing (dashed line) 
480

481

482

483

484

485

486

487

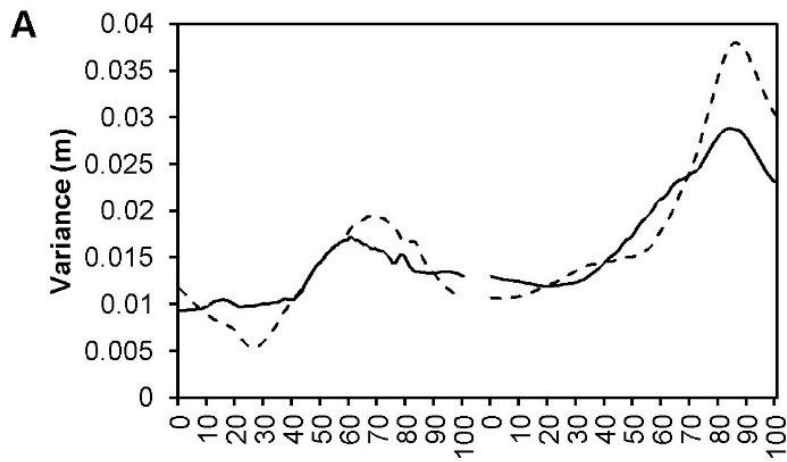

D

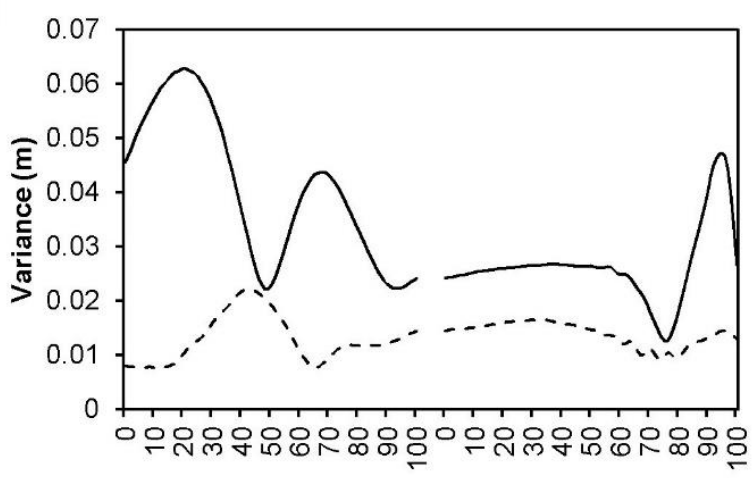

G

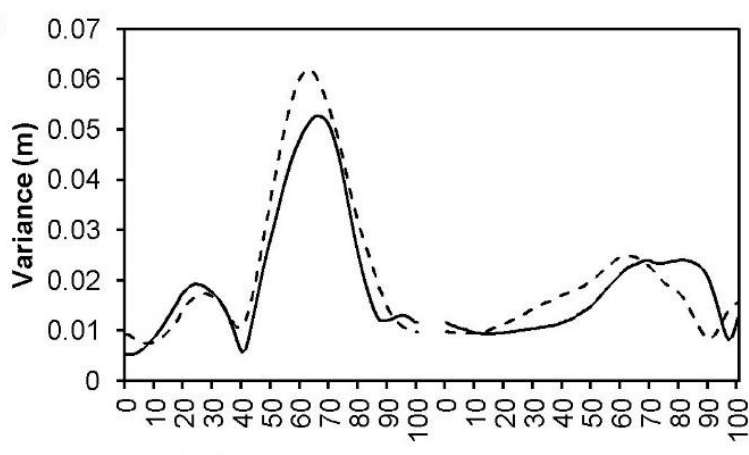

\% Backswing

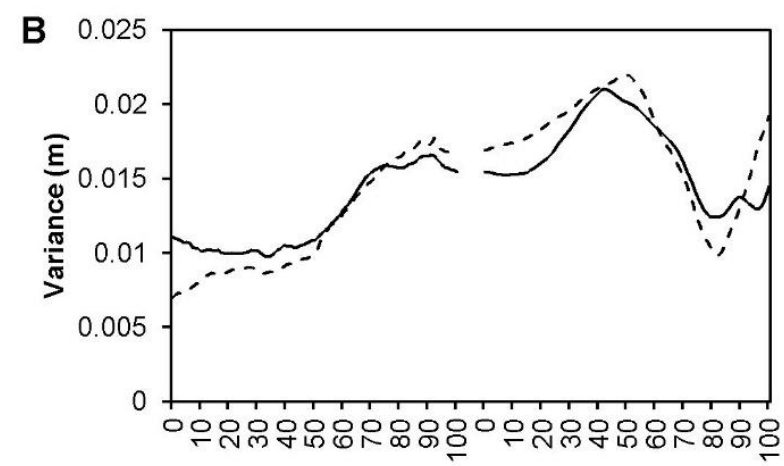

E
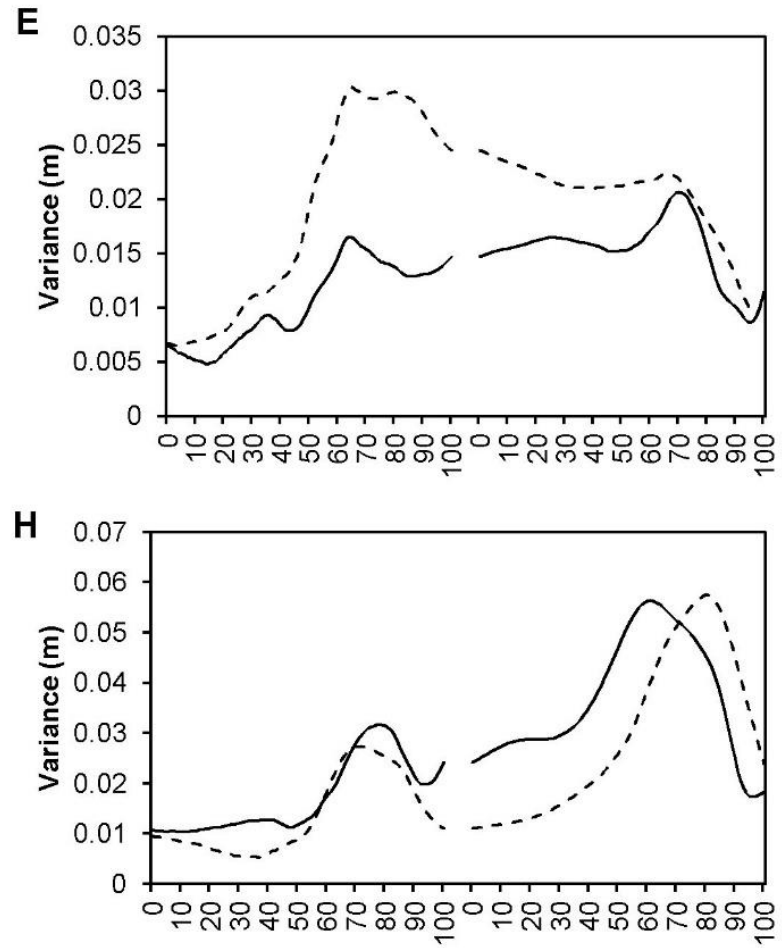

\% Backswing
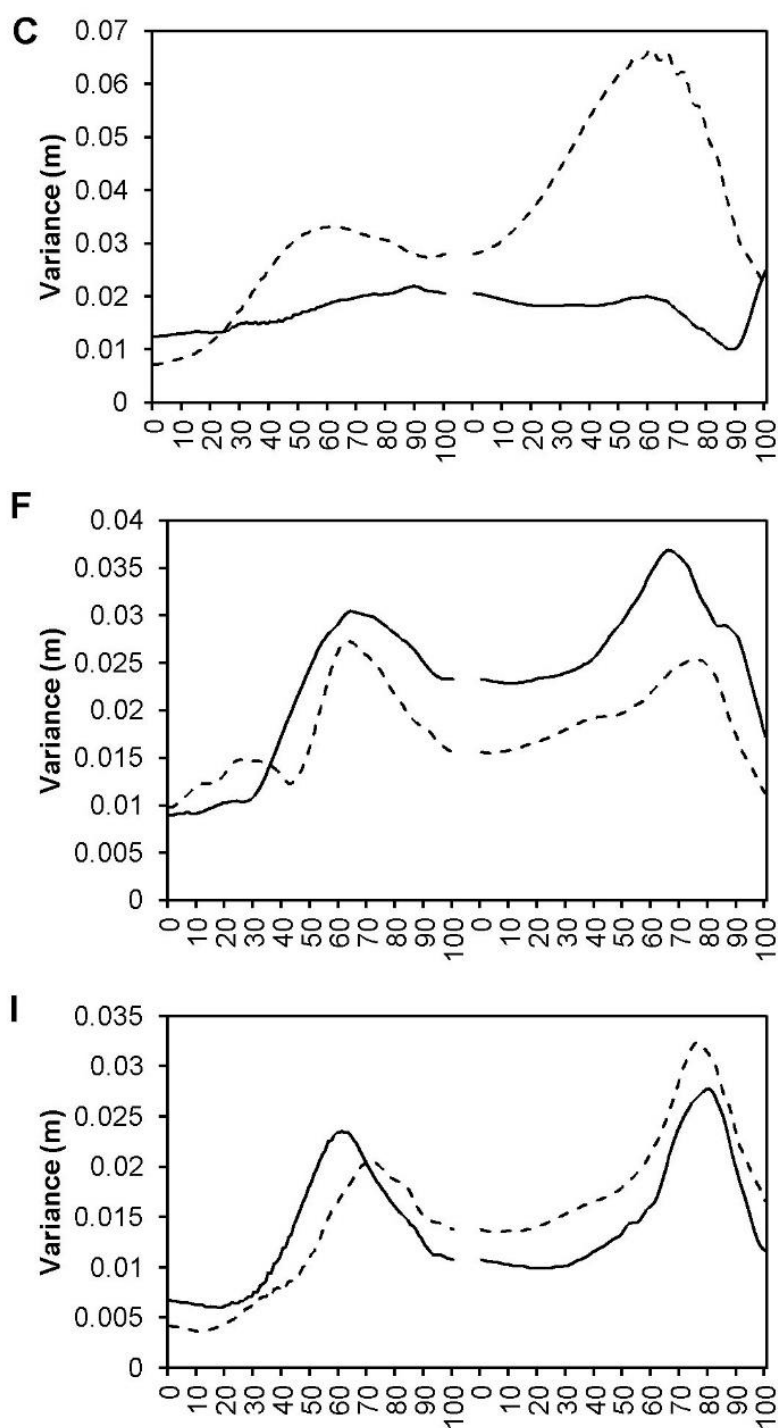

\% Backswing

$\%$ Downswing

Figure 3. Intraindividual variability of left hand's anterior-posterior position to the sternum for ball (solid line) and practice swing (dashed line) conditions. 
PRACTICE SWINGS IN GOLF

512

513

514

515

516

517

518

519

520

521

522

523

524

525

526

527

528

529

530

531

532

533

534

535

536

537

538

539

540

541

542

543

D

$\%$ Backswing conditions.
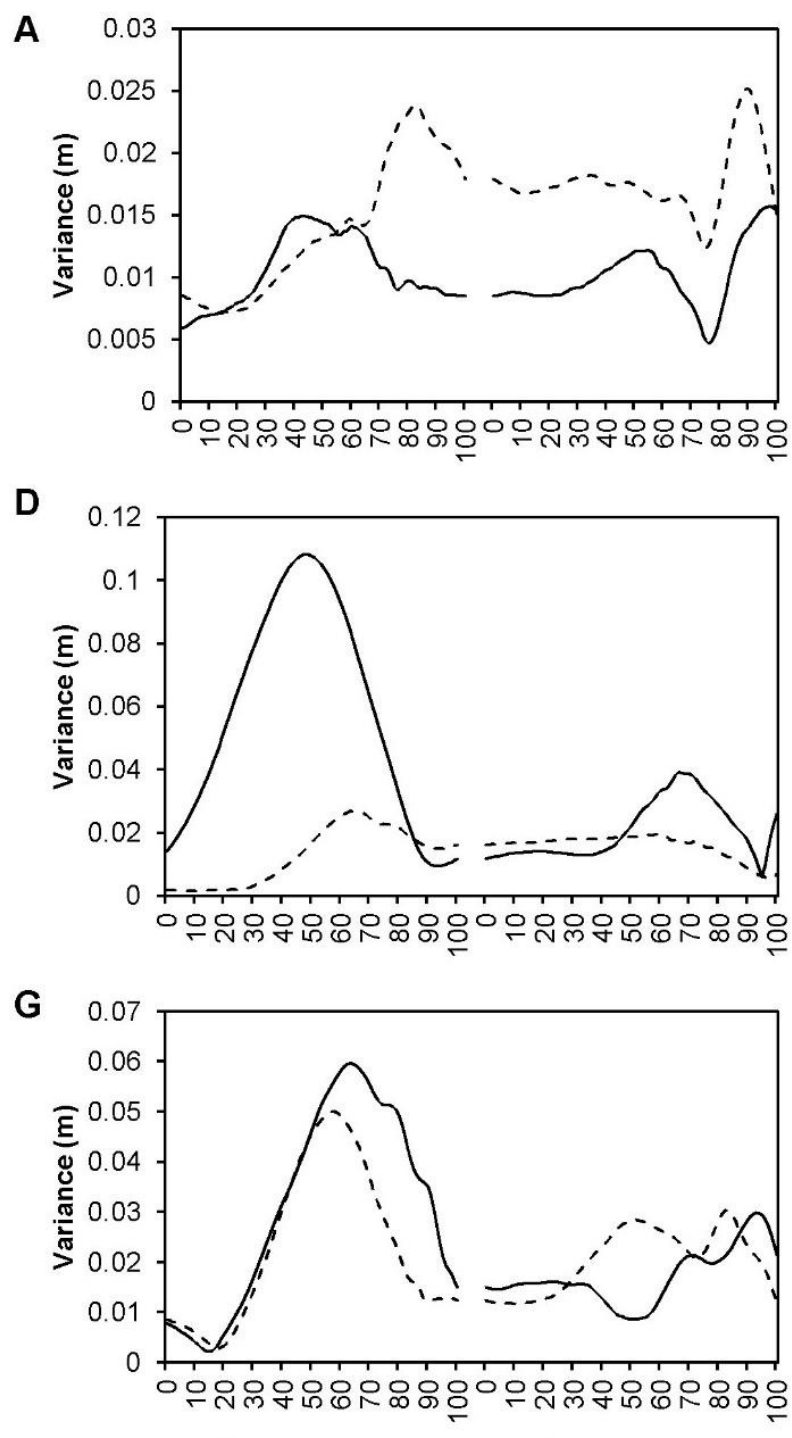

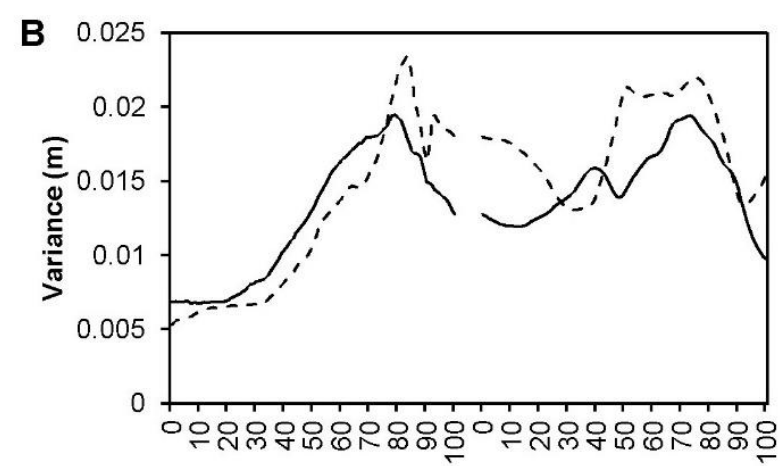

E
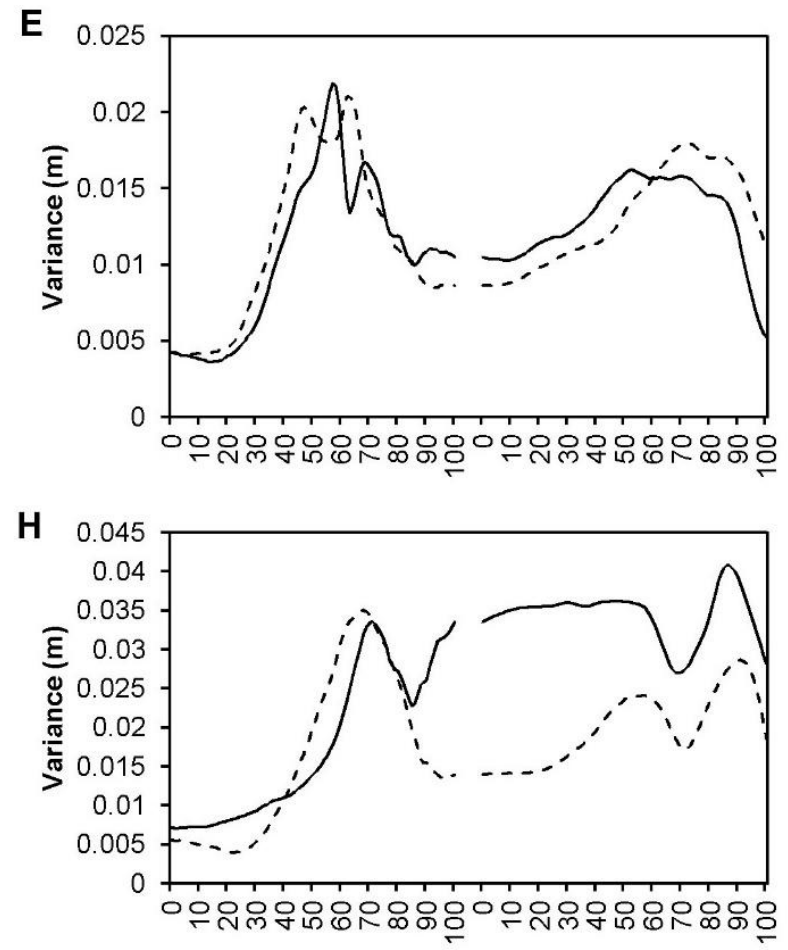

$\%$ Backswing
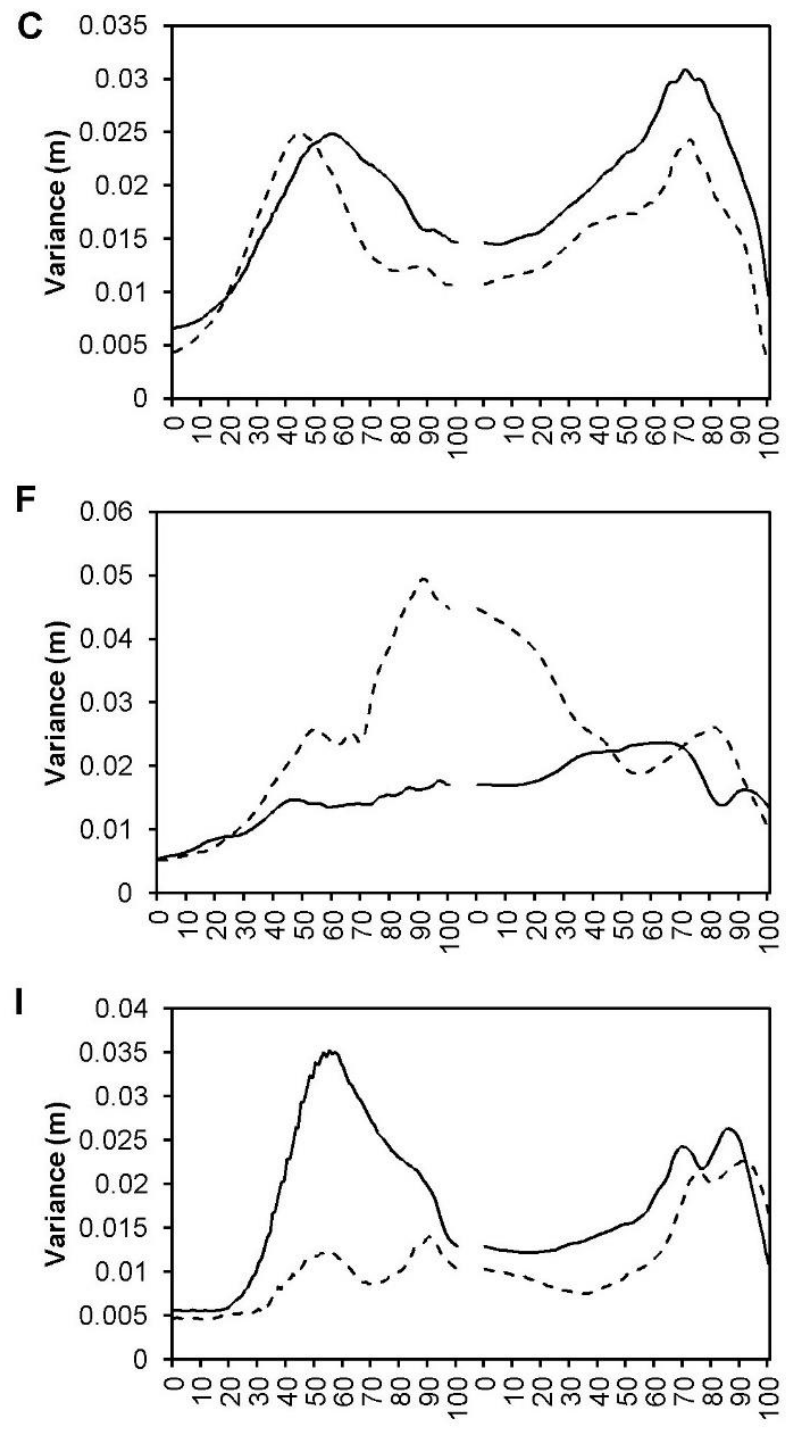

\% Backswing

\% Downswing

Figure 4. Intraindividual variability of left hand's superior-inferior position to the sternum for ball (solid line) and practice swing (dashed line) 